\title{
The Sphingosine-1-Phosphate Receptor Agonist FTY720 Increases Heart Rate Variability in Isolated Murine Ischemic Heart Model
}

\section{Emmanuel E Egom ${ }^{1-3 *}$, Thierno Madjou Bah ${ }^{4}$ and Ming Lei ${ }^{2}$}

${ }^{1}$ Hull and East Yorkshire Hospitals NHS Trust, Academic Cardiology, University of Hull, Hull, East Yorkshire HU16 5JQ, UK ${ }^{2}$ Cardiovascular Group, School of Clinical and Laboratory Sciences, The University of Manchester, Manchester, M13, 9NT UK ${ }^{3}$ Department of Physiology and Biophysics, Faculty of Medicine, Dalhousie University, Halifax, Nova Scotia, Canada

${ }^{4}$ Department of Medical Neuroscience, Faculty of Medicine, Dalhousie University, Halifax, Nova Scotia, Canada

\begin{abstract}
Despite considerable improvements in the management of hypertension, only half of adults with hypertension have their blood pressure under control. Uncontrolled hypertension may contribute to the development of left ventricular hypertrophy-induced myocardial ischemia. Accumulating evidence also suggests that hypertension may lead to lower heart rate variability (HRV), which is associated with an increased risk of cardiovascular disease. This forms the basis for the hypothesis that interventions aiming at raising HRV and reducing ischemia-induced injury in hypertensive patients, especially after AMI, may reduce the risk of cardiac events and mortality. In experiments reported here, we investigated the effects of the sphingosine-1-phosphate receptor agonist FTY720 on HRV in isolated, denervated murine ischaemic hearts. Our results demonstrated the presence of one major concentration of power centred on the ultra low frequency bands (ULF) which accounted for more than $99.9 \%$ of the total power (TP). TP and ULF decreased by $98 \%$ and $98.5 \%$ during ischemia respectively whilst these parameters increased by $1074 \%$ and $1073 \%$ during ischemia $+25 n F T Y 720$ respectively $(n=8, p<0.001)$. The Poincaré plot of the ischemia + 25 nM FTY720 condition exhibits greater dispersion of points than that of the control and ischemia conditions. The reconstructed ellipses had larger SD2 in the ischemia + 25 nM FTY720 condition than in the control and ischemia conditions ( $n=8, p<0.001$ ). These data provide the first evidence that FTY720 may increase HRV, especially after myocardial ischemia and may represent a therapeutic approach for the treatment and prevention of coronary artery disease.
\end{abstract}

Keywords: Hypertension; FTY720; Myocardial ischemia; Coronary artery disease; Myocardial ischemia

Abbreviations: HRV: Heart Rate Variability; ULF: Ultra Low Frequency; TP: Total Power

\section{Introduction}

The prevalence of hypertension among U.S. adults aged $\geq 18$ years is approximately $31 \%$, and hypertension increases with age to approximately $70 \%$ among subjects aged $\geq 65$ years [1]. Despite considerable improvements in the management of hypertension, only half of adults with hypertension have their blood pressure under control [2]. Evidence suggests that the prevalence of uncontrolled hypertension is increasing [3]. Uncontrolled hypertension may contribute to the development of left ventricular hypertrophy (LVH). In hypertrophied myocardium, there is a compression and reduced density of capillaries, and a reduced ability of the coronary arteries to dilate in response to decreased perfusion or during vasodilatory stress [4]. These factors may lead to a decreased coronary reserve and a consequent myocardial ischemia which is associated with an increased cardiovascular risk.

Evidence also suggests that hypertension is related to overall lower heart rate variability (HRV) values and low HRV values have been associated with increased mortality and the risk of cardiac events [57]. The predictive value of a depressed HRV may be independent of other known risk factors of coronary artery disease (CAD) [3]. [8]. Interestingly, improvement in HRV has been associated with a better outcome $[9,10]$. This suggests that interventions aiming at raising HRV and reducing ischemia-induced injury in hypertensive patients, especially after AMI, may represent a therapeutic approach for the treatment and prevention of CAD.

FTY720 (Fingolimod) is an orally available sphingosine-1phosphate (S1P) receptor agonist, approved by the FDA for the treatment of multiple sclerosis that induces functional change in lymphocytes and macrophages $[11,12]$. The cardiac effects of FTY720 are well known and have been described in animals and humans. Schmouder et al. demonstrated that FTY720 might have a mild to moderate negative chronotropic effect in humans [13]. Remarkably, FTY720 has been shown to significantly reduce atherosclerotic plaques in apoE deficient mice [14]. FTY720 has also been shown to both prevent ischemiareperfusion damage in isolated murine heart and sino-atrial nodes (SA) $[15,16]$. Furthermore, FTY720 reduces ischemia-induced ventricular arrhythmias and SA nodal dysfunction via activation of p21 activated kinase (Pak1), a Ser/Thr kinase downstream of small G-proteins, and Akt [15-17], consistent with prior studies in $\mathrm{S}_{1} \mathrm{P}_{2,3}$ receptor knockout mice that demonstrated an increase in infarct size following ischemia reperfusion injury [18]. On the basis of these data, we speculated that FTY720 might also increase HRV in an isolated ischemic heart preparation.

\section{Materials and Methods}

\section{Study protocol}

This study was approved by the Ethical Committee of the University

${ }^{*}$ Corresponding author: Emmanuel Eoume A Egom, Department of Physiology and Biophysics, Faculty of Medicine, Dalhousie University, Sir Charles Tupper Medical Building, Room 3F, 5850 College Street, Halifax, Nova Scotia, Canada, B3H 4R2, Tel: + 1902494 2268; Fax: + 1902494 1685; E-mail: eeegom@dal.ca

Received May 08, 2013; Accepted May 14, 2013; Published May 23, 2013

Citation: Egom EE, Bah TM, Lei M (2013) The Sphingosine-1-Phosphate Receptor Agonist FTY720 Increases Heart Rate Variability in Isolated Murine Ischemic Heart Model. Cardiol Pharmacol 2: 107. doi:10.4172/2329-6607.1000107

Copyright: ( 2013 Egom EE, et al. This is an open-access article distributed under the terms of the Creative Commons Attribution License, which permits unrestricted use, distribution, and reproduction in any medium, provided the original author and source are credited. 
Citation: Egom EE, Bah TM, Lei M (2013) The Sphingosine-1-Phosphate Receptor Agonist FTY720 Increases Heart Rate Variability in Isolated Murine Ischemic Heart Model. Cardiol Pharmacol 2: 107. doi:10.4172/2329-6607.1000107

of Manchester and the Home Office. Procedures were performed in accordance with UK national and institutional guidelines. Eight Wistar adult rats weighing $300-400 \mathrm{mg}$ were humanely killed by cervical dislocation. The excised hearts were immediately immersed in cold buffer $\left(23^{\circ} \mathrm{C}\right)$ to achieve cardioplegic arrest prior to mounting on the perfusion apparatus. The apparatus allowed coronary perfusion by Langendorff mode. In the Langendorff mode, the aortic root is retrogradely perfused, and the left ventricle performs isovolumic pressure work with a closed aortic valve; the left atrium is not filled. After a 30-minute perfusion period in Langendorff mode to stabilize the isolated heart preparation, all hearts underwent perfusion for 30 minutes with normal Tyrode solution, followed by 20 minutes with ischemic solution and finally for 30 minutes with ischemic solution plus 25 nM FTY720. Two suction electrodes for ECG recording were positioned on the right atrial and left ventricular free walls, respectively. The perfusate consisted of a modified Krebs'-Henseleit buffer containing (in $\mathrm{mM}$ ): $118 \mathrm{NaCl}, 4.7 \mathrm{KCl}, 1.2 \mathrm{MgSO}_{4}, 2 \mathrm{CaCl}_{2}$, $0.5 \mathrm{Na}_{2} \mathrm{EDTA}, 11$ glucose, $25 \mathrm{NaHCO}_{3}$, and $1.2 \mathrm{KH}_{2} \mathrm{PO}_{4}$ gassed with $95 \% \mathrm{O}_{2}-5 \% \mathrm{CO}_{2}$ to give a $\mathrm{pH}$ of 7.4. The ischemic solution and the control perfusate were similar, except for the following changes: $8 \mathrm{mM}$ $\mathrm{KCl}$, no glucose, $\mathrm{pH}$ 6.0, and bubbled with $100 \%$ nitrogen gas for more than $45 \mathrm{~min}$ before the experiment was started. Temperature was set at $36^{\circ} \mathrm{C}$ at the inner surface of the right ventricular apex. ECG data were digitized at a sampling rate of $300 \mathrm{~Hz}$ and stored for subsequent off-line data analysis.

\section{Analysis of HRV}

The HRV analysis was performed by measuring the frequency domain and the Poincare plot indexes. The ECG was continuously monitored. Spectral analysis was done with Fast Fourier Transformation algorithms using a commercial software kit (MATLAB, Mathworks, Inc., Natick, MA, USA). To obtain the indexes, the area under different bands of the power spectrum was measured. Five measures of HRV in the frequency domain were computed (Table 1). The Poincaré plot is a diagram in which each R-R interval of a tachogram or HRV time series is plotted as a function of the previous $\mathrm{R}-\mathrm{R}$ interval. We used pairs of R-R intervals lagged by one, two, and four beats. The quantitative analysis of the Poincaré plot is based on the notion that each R-R interval is influenced by previous vagal and sympathetic modulations over the heart rate and therefore the pairs of successive R-R intervals form an attractor in the Poincaré plot. An ellipse was adjusted to the Poincaré plot attractor after calculation of the minor and the major axes as follows: the Poincaré plot is first turned $45^{\circ}$ counter clockwise and the standard descriptors (SD) of the data along the horizontal axis (SD1 or minor axis) is then computed. SD1 shows the SD of the instantaneous beat-to-beat variability of the data. The SD of the continuous long-term $\mathrm{R}-\mathrm{R}$ intervals (SD2 or major axis) is measured along the horizontal axis, after the plot is turned $45^{\circ}$ clockwise. The point where both axes intersect corresponds to the total mean of the R-R intervals [19].

\begin{tabular}{|l|l|l|}
\hline \multicolumn{1}{|c|}{ Variables } & \multicolumn{1}{c|}{ Unit } & \multicolumn{1}{c|}{ Definition } \\
\hline Mean R-R & $\mathrm{ms}$ & Mean RR interval \\
\hline Total power (TP) & $\mathrm{ms}^{2}$ & Approximately $\leq 0.4 \mathrm{~Hz}$ \\
\hline Ultra low frequency & $\mathrm{ms}^{2}$ & Approximately $\leq 0.003 \mathrm{~Hz}$ \\
\hline domain (ULF) & & \\
\hline Very low frequency & $\mathrm{ms}^{2}$ & Power from 0.003 to $0.04 \mathrm{~Hz}$ \\
\hline Domain (VLF) & & \\
\hline Low frequency domain (LF) & $\mathrm{ms}^{2}$ & Power from 0.04 to $0.15 \mathrm{~Hz}$ \\
\hline High frequency domain (HF) & $\mathrm{ms}^{2}$ & Power from 0.15 to $0.4 \mathrm{~Hz}$ \\
\hline
\end{tabular}

Table 1 1 Measures of heart rate variability in the frequency domain.

\section{Statistical analysis}

All data are reported as means \pm S.D. Repeated measure oneway ANOVA analysis of variance was used to compare values of measurements obtained from the heart preparation. When analysis of variance revealed the existence of a significant difference among values, Tukey's test was applied to determine the significance of a difference between selected group means. A p value $<0.05$ was taken as an upper limit to indicate a significant difference.

\section{Results}

\section{Effect of FTY720 on ischemia-induced bradycardia}

We first studied the effect of FTY720 on ischemia-induced bradycardia in Langendorff ex vivo heart. The ex vivo hearts were first perfused with normal Tyrode solution for $30 \mathrm{~min}$ and then exposed to global ischemia by perfusion with the ischemic solution for $20 \mathrm{~min}$ and, finally, followed by $30 \mathrm{~min}$ of ischemia in the presence of $25 \mathrm{nM}$ of FTY720. As shown in figure 1, RR interval increased by $69 \%$ during ischemia and by $24 \%$ during ischemia +25 nM FTY720 from baseline (control: $230 \pm 17 \mathrm{~ms}$; ischemia: $741 \pm 138 \mathrm{~ms}$; ischemia $+25 \mathrm{nM}$ FTY720: $302 \pm 121, \mathrm{n}=8, \mathrm{p}<0.001)$. Thus, FTY720 attenuated ischemiainduced bradycardia.

\section{Power spectral analysis}

The Langendorff ex vivo heart model preparations were also used to study the effect of FTY720 on HRV under ischemia condition. The ex vivo hearts were also either perfused with normal Tyrode solution or equilibrated for $30 \mathrm{~min}$ and then exposed to global ischemia by perfusion with the ischaemic solution for $20 \mathrm{~min}$. Heart rate was recorded for $2 \mathrm{~h}$ for each of eight animals. A Fast Fourier transform was used to derive the HRV power spectrum. Changes in frequency and amplitude of the various spectral peaks were quantitatively examined. In figure 2 typical examples of HRV time series of control, ischemia and ischemia $+25 \mathrm{nM}$ FTY720 conditions are shown. We found that the percentage of total power values for the ULF band of HRV frequency spectrum was $99.94 \%$ suggesting that most of the HRV on isolated denervated murine heart is related to this band of spectrum (TP, $2271580 \pm 557098$ vs. $2270230 \pm 537057, \mathrm{ULF}$ ). As summarized in table 2 , total power and ULF decreased by $98 \%$ and $98.5 \%$ during

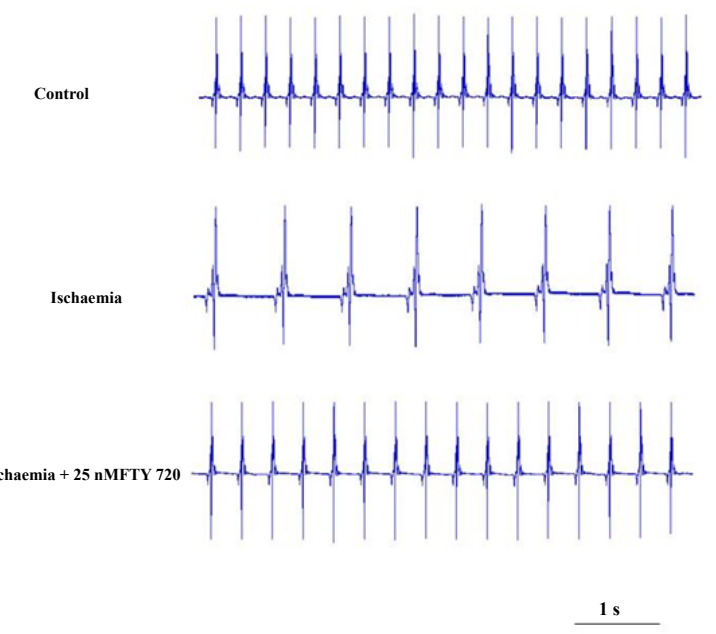

Figure 1 ECG recordings showing the effect of FTY720 on ischemia-induced bradycardia in rat heart preparations. Ischemia reduced $R R$ interval and this effect was blunted by a co-administration of 25 nM of FTY720 $(n=8 ; P<0.001)$. 


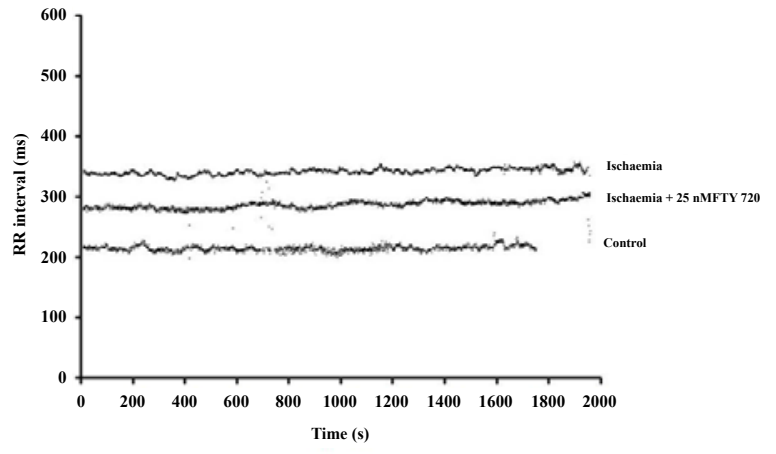

Figure $2 \square R-R$ interval tachograms from control $(A)$; ischemia $(B)$ and ischemia + FTY720 (C).

\begin{tabular}{|l|l|l|l|l|}
\hline Variable & Unit & \multicolumn{1}{|c|}{ Control } & \multicolumn{1}{|c|}{ Ischemia } & \multicolumn{1}{c|}{ Ischemia+FTY720 } \\
\hline Mean RR & $\mathrm{ms}$ & $230 \pm 17$ & $741 \pm 138^{*}$ & $302 \pm 121 \pm \S$ \\
\hline TP & $\mathrm{ms}^{2}$ & $2271580 \pm 557098$ & $51492 \pm 11905^{*}$ & $26662929 \pm 6768702 \pm \S$ \\
\hline ULF & $\mathrm{ms}^{2}$ & $2270230 \pm 537057$ & $34519 \pm 9105^{*}$ & $26634969 \pm 659792 \ddagger \S$ \\
\hline VLF & $\mathrm{ms}^{2}$ & $993 \pm 278$ & $12 \pm 28^{*}$ & $20008 \pm 4980 \pm \S$ \\
\hline LF & $\mathrm{ms}^{2}$ & $142 \pm 83$ & $26 \pm 12^{*}$ & $3170 \pm 1405 \pm \S$ \\
\hline HF & $\mathrm{ms}^{2}$ & $108 \pm 60$ & $10 \pm 6^{*}$ & $951 \pm 473 \pm \S$ \\
\hline
\end{tabular}

Values: mean $\pm \mathrm{SD}$. ${ }^{*} \mathrm{P}<0.001$ for the Mean RR, TP, ULF, VLF, LF and HF comparison between the control vs. ischemia. $\ddagger P<0.001$ for the Mean RR, TP, ULF, VLF, LF and HF comparison between the control vs. ischemia + FTY720. $\S \mathrm{P}<0.001$ for the Mean RR, TP, ULF, VLF, LF and HF comparison between the ischemia vs. ischemia + FTY720.

Table 2 $\square$ Heart Rate Variability analysed in the Frequency Domain in the isolated, denervated rat heart during control and ischemia conditions.

ischemia respectively whilst these parameters increased by $1074 \%$ and $1073 \%$ during ischemia $+25 n$ FTY720 respectively $(n=8, p<0.001)$. The remaining frequency domain or power spectral measures of HRV such as VLF, LF, HF were found to be substantially reduced or abolished on both control and ischemia conditions respectively $(\mathrm{n}=8, \mathrm{p}<0.001)$. Figure 3 shows global results from the statistical and power spectral analysis in control, ischemia and ischemia $+25 \mathrm{~nm}$ FTY720 conditions.

\section{Poincaré analysis}

Some evidence suggests that qualitative analysis of Poincaré (or Lorenz) plot patterns may identify higher risk CAD patients in whom standard time and frequency domain HRV measures are not different [20]. Other data also suggest Poincaré plot analysis might also provide prognostic information that is independent of and complementary to traditional frequency domain HRV [21]. Thus, Poincaré plot was also used to evaluate HRV. Each R-R interval was plotted against the previous one so that the coordinates of each point are R-R and R-R1. An ellipse fitted to the Poincaré plot was used to provide numeric measures of their magnitude. The short axis of the fitted ellipse was designated as SD1 and the long one as SD2. The ratio was then called SD1/2. Typical Poincaré plots are shown in figure 4. As the plot gets more abnormal, the short axis tends to get larger relative to the long one and SD1/2 becomes larger as a result. Table 3 shows the Poincaré plot indexes measured from Poincaré plots. As summarized in table 3 , SD2 was significantly greater in the ischemia $+25 \mathrm{nM} \mathrm{FTY720}$ condition than in the control and ischemia conditions $(\mathrm{n}=8, \mathrm{p}<0.001)$. No statistically significant differences were detected by the Poincaré indexes among other conditions but there was a trend toward an increase and a decrease of SD1/2 in ischemia and ischemia $+25 \mathrm{nM}$ FTY720 conditions respectively compared with the control.

\section{Discussion}

Our data are the first to demonstrate that the $\mathrm{S} 1 \mathrm{P}$ receptor agonist FTY720 can increase HRV. These data also significantly extend earlier reports providing evidence for a role of FTY720 in cardiac protection as FTY720 effectively antagonized ischemia-induced bradycardia. Moreover, we also found that HRV was significantly reduced during ischemia and FTY720 increased HRV as the Poincare plot of the ischemia +25 nM FTY720 condition exhibited greater dispersion of points than that of the Control and ischemia conditions. The reconstructed ellipses had larger SD2 in the ischemia + 25 nM FTY720 condition than in the control and ischemia conditions. There was also a trend toward an increase and a decrease of SD1/2 in ischemia and ischemia +25 nM FTY720 conditions respectively compared with the control.

We also demonstrated the presence of one major concentration of power centred on ULF $(\leq 0.003 \mathrm{~Hz})$. ULF power accounted for more than $99.9 \%$ of the total power. Although the pattern of HRV was of random, broad-band fluctuations, we observed variations in this spectral peak, especially its amplitude, both during ischemia and ischemia + 25 nM FTY720 phases. The amplitude of this spectral peak decreased and increased significantly in ischemia and ischemia +25 nM FTY720 respectively. The remaining frequency domain or power spectral measures of HRV such as VLF, LF, and HF were found to be substantially reduced or abolished on both control and ischemia conditions respectively. This is likely to be due to the denervation as the experiments were conducted on isolated denervated murine heart. In consistence with our results, Raeder and colleagues observed that surgical denervation of the heart in the anesthetized dog immediately abolished most heart rate fluctuations in the $0.02-$ to $0.3-\mathrm{Hz}$ frequency bands [22]. Moreover, there is considerable evidence that the power in the HF band is a function of variation in parasympathetic nervous system activity, and that modulation of both parasympathetic and sympathetic activity contributes to the LF band [23-26].

Evidence suggests that hypertension is related to overall lower HRV values and may be associated with myocardial ischemia [6,7]. Declines in most HRV measures, although not HF power, were seen in those who suffered myocardial ischemia during that period [27]. In a direct comparison of data from a large post-AMI study and data from healthy age- and gender-matched normal controls, six power spectral measures of HRV (ULF, VLF, LF, HF, total power, and LF/HF ratio) were found to be substantially reduced 10 days after AMI [28]. In a canine model of AMI, coronary artery ligation is followed by an abrupt decrease in HRV [29].

A depressed HRV has an important clinical relevance as it has also been associated with both arrhythmic and non-arrhythmic cardiac death suggesting that HRV in combination with other risk factors for arrhythmic death may help identify high-risk patients [30]. In fact, one

\begin{tabular}{|l|l|l|l|}
\hline \multicolumn{1}{|c|}{ Variable } & \multicolumn{1}{c|}{ Unit } & \multicolumn{1}{c|}{ Control Ischemia } & \multicolumn{1}{c|}{ Ischemia+FTY720 } \\
\hline SD1 & $\mathrm{ms}$ & $5.9 \pm 44.3 \pm 3$ & $5 \pm 2.5$ \\
\hline SD2 & $\mathrm{ms}$ & $19.6 \pm 111.5 \pm 1^{*}$ & $41 \pm 2 \ddagger \S$ \\
\hline SD1/SD2 & $\mathrm{N} / \mathrm{A}$ & $0.3 \pm 0.20 .38 \pm 0.3$ & $0.13 \pm 0.1$ \\
\hline
\end{tabular}

Values are means \pm standard error; SD1 = standard deviation of instantaneous beat-to-beat variability; SD2 = standard deviation of long-term continuous variability; SD1/SD2 = ratio between instantaneous and long-term standard deviation. ${ }^{*} \mathrm{P}<0.001$ for the SD2 comparison between the control vs. ischemia $\ddagger \mathrm{P}<0.001$ for the SD2 comparison between the control vs. ischemia + FTY720. $\S \mathrm{P}<0.001$ for the SD2 comparison between the ischemia vs. ischemia + FTY720.

Table 3₫ Quantitative Two-Dimensional Analysis of Poincaré Plots of Successive RR Intervals. 

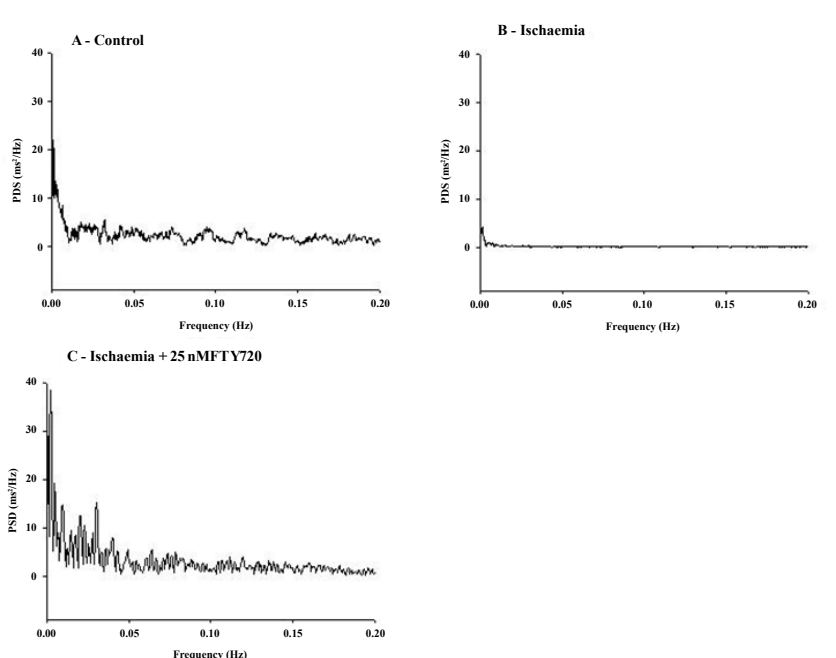

Figure 3] Power spectrum analysis of an isolated denervated rat heart preparation from control (A); ischemia (B) and ischemia + FTY720 (C). Power spectrum density is shown as a semilogarithmic plot.

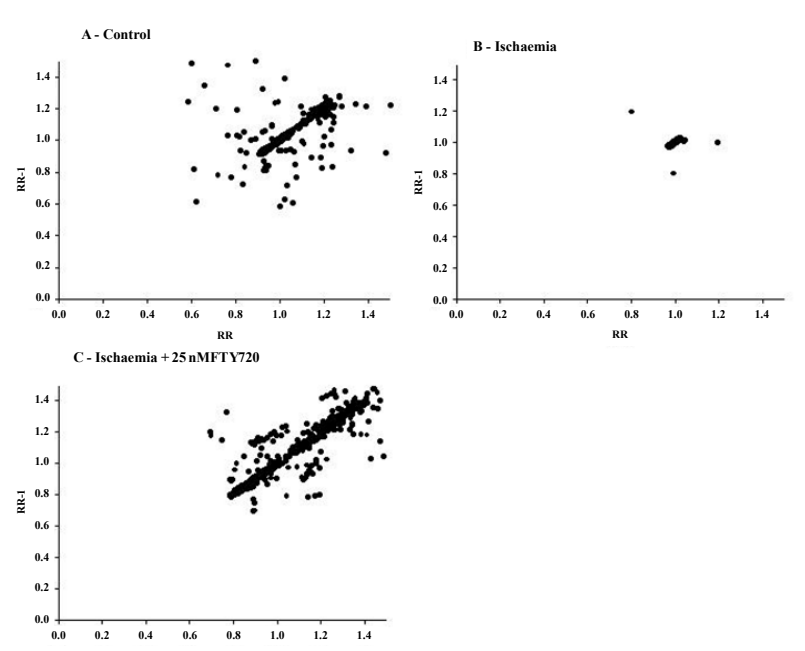

Figure $4 \square$ Poincaré plots of R-R intervals. A) Control. B) Effect of ischemia on HRV. C) Effect of ischemia on HRV in the presence of 25 nM FTY720.

of the proven clinical uses of HRV is the prediction of risk cardiac death or arrhythmic events after AMI [23]. The first large study to show the usefulness of HRV for predicting long-term outcome after AMI, the Multi-Center Post-Infarction Program, or MPIP, involved 808 patients who underwent ambulatory monitoring within 11 days of AMI [31]. After a mean 31-months follow-up, HRV measures predicted mortality independently of other risk predictors such as left ventricular ejection fraction or ventricular arrhythmias. In a reanalysis of the MPIP data, after a four-year follow-up, all frequency domain measures of HRV were associated with all cause, cardiac, and arrhythmic death; the strongest univariate association was for total power and ULF power. After adjustment for five established risk predictors (age, NYHA functional class, rales detected on chest auscultation in the coronary care unit, left ventricular ejection fraction, and ventricular arrhythmias), the association between mortality and total power, ULF power remained significant whereas VLF, LF power and HF power were only moderately strongly associated with mortality [32]. The continuing validity of HRV for prediction of outcome post-AMI was confirmed in the GISSI-2 study of fibrinolytic therapy in AMI [33]. A subset of 567 patients had a valid 24-hour ambulatory ECG recording and 52 of them died during 1000 days of follow-up. Decreased values of HRV identified high risk groups comprising 16 to 18 percent of the cohort, with mortalities ranging from 20.8 to 24.2 percent in the high-risk group versus 6.0 to 6.8 percent in the low-risk group. Thus, the relative risk of mortality was approximately 3.0 for the low HRV groups. The largest recent study of the predictive value of HRV post-MI was ATRAMI (Autonomic Tone and Reflexes after Myocardial Infarction) [34]. Patients (N=1284) had Holter recordings 28 days post-MI and were followed for $21 \pm 8$ months. Depressed HRV was associated with a relative risk of mortality of 3.2. A meta-analysis of 21 studies with 3489 patients confirmed the ATRAMI findings, showing that patients with depressed HRV (8.1 percent of patients) were almost four times as likely to die over the following three years [35].

Although the prognostic value of HRV relies heavily on changes in ULF power, the physiologic correlates of ULF bands are not established [23-26,36-40]. Suggested causes of heart rate variability in the ULF bands include variations in hormones of the renin-angiotensin-aldosterone system, temperature regulation, circulating catecholamines, or changes in physical activity [24,41-44].

As demonstrated in experiments reported here and consistent with other reports, most of the spectral power is contained in this lowest frequency band [37]. As a decreased ULF power is a powerful predictor of death in the years after AMI, mechanisms regulating ULF bands may have therapeutic implications: agents that can increase a HRV at the ULF (as shown here with FTY720) may have therapeutic value after infarction, particularly in patients with low HRV [37].

\section{Conclusion}

In conclusion, we have provided the first evidence that FTY720 increases HRV in an ischaemic murine model. The present study also suggests that a decreased HRV during ischemia in isolated heart preparation is mainly at the ULF. In view of the powerful and independent prediction of mortality of this component of the heart period power spectrum, interventions aiming at raising HRV at the ULF in post-AMI hypertensive patients, such as FTY720, may represent a therapeutic approach for the treatment and prevention of CAD.

\section{Acknowledgement}

EEE is a recipient of the Heart and Stroke Foundation of Canada Fellowship. The project was supported by The Welcome Trust (ML) and The British Heart Foundation (ML).

\section{References}

1. CDC (2011) Vital signs: prevalence, treatment, and control of hypertensionUnited States, 1999-2002 and 2005-2008. MMWR 60: 103-108.

2. Yoon SS, Ostchega Y, Louis T (2010) Recent trends in the prevalence of high blood pressure and its treatment and control, 1999--2008. NCHS Data Brief no 482010.

3. Ma J, Lee KV, Stafford RS (2006) Changes in antihypertensive prescribing during US outpatient visits for uncomplicated hypertension between 1993 and 2004. Hypertension 48: 846-852.

4. Beache GM, Herzka DA, Boxerman JL, Post WS, Gupta SN, et al. (2001) Attenuated myocardial vasodilator response in patients with hypertensive hypertrophy revealed by oxygenation-dependent magnetic resonance imaging Circulation 104: 1214-1217.

5. Tsuji H, Larson MG, Venditti FJ Jr, Manders ES, Evans JC, et al. (1996) Impact of reduced heart rate variability on risk for cardiac events. The Framingham Heart Study. Circulation 94: 2850-2855.

6. Schroeder EB, Liao D, Chambless LE, Prineas RJ, Evans GW, et al. (2003) 
Citation: Egom EE, Bah TM, Lei M (2013) The Sphingosine-1-Phosphate Receptor Agonist FTY720 Increases Heart Rate Variability in Isolated Murine Ischemic Heart Model. Cardiol Pharmacol 2: 107. doi:10.4172/2329-6607.1000107

Page 5 of 5

Hypertension, blood pressure, and heart rate variability: the Atherosclerosis Risk in Communities (ARIC) study. Hypertension 42: 1106-1111.

7. Singh JP, Larson MG, Tsuji H, Evans JC, O'Donnell CJ, et al. (1998) Reduced heart rate variability and new-onset hypertension: insights into pathogenesis of hypertension: the Framingham Heart Study. Hypertension 32: 293-297.

8. Ponikowski P, Anker SD, Chua TP, Szelemej R, Piepoli M, et al. (1997) Depressed heart rate variability as an independent predictor of death in chronic congestive heart failure secondary to ischemic or idiopathic dilated cardiomyopathy. Am J Cardiol 79: 1645-1650.

9. Fantoni C, Raffa S, Regoli F, Giraldi F, La Rovere MT, et al. (2005) Cardiac resynchronization therapy improves heart rate profile and heart rate variability of patients with moderate to severe heart failure. J Am Coll Cardiol 46: 18751882.

10. Landolina M, Gasparini M, Lunati M, Santini M, Rordorf R, et al. (2008) Heart rate variability monitored by the implanted device predicts response to CRT and long-term clinical outcome in patients with advanced heart failure. Eur $\mathrm{J}$ Heart Fail 10: 1073-1079.

11. Cohen JA, Barkhof F, Comi G, Hartung H-P, Khatri BO, et al. (2010) Ora Fingolimod or Intramuscular Interferon for Relapsing Multiple Sclerosis. N Engl J Med 362: 402-415.

12. Kappos L, Radue E-W, O'Connor P, Polman C, Hohlfeld R, et al. (2010) A Placebo-Controlled Trial of Oral Fingolimod in Relapsing Multiple Sclerosis. N Engl J Med 362: 387-401.

13. Schmouder R, Serra D, Wang Y, Kovarik JM, DiMarco J, et al. (2006) FTY720: Placebo-Controlled Study of the Effect on Cardiac Rate and Rhythm in Healthy Subjects. The Journal of Clinical Pharmacology 46: 895-904.

14. Keul P, Tolle M, Lucke S, von Wnuck Lipinski K, Heusch G, et al. (2007) The sphingosine-1-phosphate analogue FTY720 reduces atherosclerosis in apolipoprotein E-deficient mice. Arterioscler Thromb Vasc Biol 27: 607-613.

15. Egom EE, Ke Y, Solaro RJ, Lei M (2010) Cardioprotection in ischemia/ reperfusion injury: spotlight on sphingosine-1-phosphate and bradykinin signalling. Prog Biophys Mol Biol 103: 142-147.

16. Egom EE, Mamas MA, Clark AL (2012) The potential role of sphingolipidmediated cell signaling in the interaction between hyperglycemia, acute myocardial infarction and heart failure. Expert Opin Ther Targets 16: 791-800.

17. Egom EE, Ke Y, Musa H, Mohamed TM, Wang T, et al. (2010) FTY720 prevents ischemia/reperfusion injury-associated arrhythmias in an ex vivo rat heart model via activation of Pak1/Akt signaling. J Mol Cell Cardiol 48: 406-414.

18. Means CK, Brown JH (2009) Sphingosine-1-phosphate receptor signalling in the heart. Cardiovasc Res 82: 193-200.

19. Tulppo MP, Makikallio TH, Takala TE, Seppanen T, Huikuri HV (1996) Quantitative beat-to-beat analysis of heart rate dynamics during exercise. Am J Physiol 271: H244-H252.

20. Woo MA, Stevenson WG, Moser DK, Middlekauff HR (1994) Complex heart rate variability and serum norepinephrine levels in patients with advanced heart failure. J Am Coll Cardiol 23: 565-569.

21. Stein PK, Le Q, Domitrovich PP (2008) Development of more erratic heart rate patterns is associated with mortality post-myocardial infarction. J Electrocardiol 41: $110-115$

22. Raeder EA, Berger RD, Kenet R (1987) Assessment of autonomic cardiac control by power spectrum of heart rate fluctuations. J Appl Cardiol 2: 283-300.

23. Heart rate variability: standards of measurement, physiological interpretation and clinical use. Task Force of the European Society of Cardiology and the North American Society of Pacing and Electrophysiology. Circulation 93: 10431065.

24. Akselrod S, Gordon D, Ubel FA, Shannon DC, Berger AC, et al. (1981) Power spectrum analysis of heart rate fluctuation: a quantitative probe of beat-to-beat cardiovascular control. Science 213: 220-222.

25. Yamamoto $Y$, Hughson RL (1994) On the fractal nature of heart rate variability in humans: effects of data length and beta-adrenergic blockade. Am J Physio 266: R40-R49.

26. Yamamoto Y, Nakamura Y, Sato H, Yamamoto M, Kato K, et al. (1995) On the fractal nature of heart rate variability in humans: effects of vagal blockade. Am J Physiol 269: R830-R837.

27. Bjorkander I, Forslund L, Ericson M, Rehnqvist N, Hjemdahl P, et al. (2009)
Long-term stability of heart rate variability in chronic stable angina pectoris, and the impact of an acute myocardial infarction. Clin Physiol Funct Imaging 29: $201-208$

28. Bigger JT Jr, Fleiss JL, Steinman RC, Rolnitzky LM, Schneider WJ, et al. (1995) RR variability in healthy, middle-aged persons compared with patients with chronic coronary heart disease or recent acute myocardial infarction. Circulation 91: 1936-1943.

29. Hull SS Jr, Evans AR, Vanoli E, Adamson PB, Stramba-Badiale M, et al. (1990) Heart rate variability before and after myocardial infarction in conscious dogs at high and low risk of sudden death. J Am Coll Cardiol 16: 978-985

30. Farrell TG, Bashir Y, Cripps T, Malik M, Poloniecki J, et al. (1991) Risk stratification for arrhythmic events in postinfarction patients based on heart rate variability, ambulatory electrocardiographic variables and the signal-averaged electrocardiogram. J Am Coll Cardiol 18: 687-697.

31. Kleiger RE, Miller JP, Bigger JT Jr, Moss AJ (1987) Decreased heart rate variability and its association with increased mortality after acute myocardial infarction. Am J Cardiol 59: 256-262.

32. Bigger JT Jr., Fleiss JL, Steinman RC, Rolnitzky LM, Kleiger RE, et al. (1992) Correlations among time and frequency domain measures of heart period variability two weeks after acute myocardial infarction. Am J Cardiol 69: 891 898

33. Zuanetti G, Neilson JM, Latini R, Santoro E, Maggioni AP, et al. (1996) Prognostic significance of heart rate variability in post-myocardial infarction patients in the fibrinolytic era. The GISSI-2 results. Gruppo Italiano per lo Studio della Sopravvivenza nell' Infarto Miocardico. Circulation 94: 432-436.

34. La Rovere MT, Bigger JT Jr, Marcus FI, Mortara A, Schwartz PJ (1998) Baroreflex sensitivity and heart-rate variability in prediction of total cardiac mortality after myocardial infarction. ATRAMI (Autonomic Tone and Reflexes after Myocardial Infarction) Investigators. Lancet 351: 478-484.

35. Buccelletti E, Gilardi E, Scaini E, Galiuto L, Persiani R, et al. (2009) Heart rate variability and myocardial infarction: systematic literature review and metanalysis. Eur Rev Med Pharmacol Sci 13: 299-307.

36. Bigger JT Jr, Fleiss JL, Rolnitzky LM, Steinman RC (1993) Frequency domain measures of heart period variability to assess risk late after myocardial infarction. J Am Coll Cardiol 21: 729-736.

37. Bigger JT Jr, Fleiss JL, Steinman RC, Rolnitzky LM, Kleiger RE, et al. (1992) Frequency domain measures of heart period variability and mortality after myocardial infarction. Circulation 85: 164-171.

38. Fei L, Copie X, Malik M, Camm AJ (1996) Short- and long-term assessment of heart rate variability for risk stratification after acute myocardial infarction. Am J Cardiol 77: 681-684.

39. Huikuri HV Niemela MJ, Ojala S, Rantala A Ikaheimo MJ, et al (1994) Circadian rhythms of frequency domain measures of heart rate variability in healthy subjects and patients with coronary artery disease. Effects of arousal and upright posture. Circulation 90: 121-126.

40. Tsuji H, Venditti FJ Jr, Manders ES, Evans JC, Larson MG, et al. (1994) Reduced heart rate variability and mortality risk in an elderly cohort. The Framingham Heart Study. Circulation 90: 878-883.

41. Bernardi L, Valle F, Coco M, Calciati A, Sleight P (1996) Physical activity influences heart rate variability and very-low-frequency components in Holter electrocardiograms. Cardiovasc Res 32: 234-237.

42. Dela F, Mikines KJ, Von Linstow M, Galbo H (1992) Heart rate and plasma catecholamines during $24 \mathrm{~h}$ of everyday life in trained and untrained men. $J$ Appl Physiol 73: 2389-2395.

43. Fleisher LA, Frank SM, Sessler DI, Cheng C, Matsukawa T, et al. (1996) Thermoregulation and heart rate variability. Clin Sci (Lond) 90: 97-103.

44. Osterhues HH, Hanzel SR, Kochs M, Hombach V (1997) Influence of physica activity on 24-hour measurements of heart rate variability in patients with coronary artery disease. Am J Cardiol 80: 1434-1437. 